Trauma Berufskrankh 2018 20 (Suppl 1):S1-S5 DOI 10.1007/s10039-017-0269-3

Online publiziert: 21. Juni 2017

c) Springer Medizin Verlag GmbH 2017

CrossMark

\title{
M. Kertai
}

Kinderchirurgie und Kinderorthopädie, Klinik St. Hedwig, Krankenhaus Barmherzige Brüder Regensburg, Regensburg, Deutschland

\section{Behandlung der Femurschaftfraktur im Wachstumsalter}

\section{Vom Beckenbeingips bis zur elastisch stabilen intramedullären Nagelung}

Knochen. Daher kommen Femurschaftfrakturen beim Kleinkind in der Regel auch als Monotrauma bei verhältnismäßig kleinen Krafteinwirkungen vor, während sie bei älteren Kindern analog zum Erwachsenenalter Verletzungen im Rahmen von Hochrasanztraumen sind und entsprechend häufig Begleitverletzungen aufweisen [4].

Unter den Frakturen des Femurschaftes des ersten Altersgipfels, also der Kleinkinder, spielen 2 Frakturmechanismen, neben den typischen Unfällen, beim Spielen eine wichtige Rolle: Zum einen stellt die Femurschaftfraktur nach der Klavikulafraktur die zweithäufigste geburts- traumatische Fraktur eines Röhrenknochens dar, zum anderen sind 15-80\% der Frakturen bei noch nicht gehfähigen Kindern auf eine Kindesmisshandlung zurückzuführen, wobei sich die große statistische Breite durch die hohe Dunkelziffer erklären lässt [5,6]. Daher gelten alle Femurschaftfrakturen bei nicht gehfähigen Kindern primär als misshandlungsverdächtig und benötigen eine entsprechende Abklärung bzw. die Identifikation einer Differenzialdiagnose, wie z. B. einer Osteogenesis imperfecta.

In allen Altersklassen kommen $\mathrm{Fe}$ murschaftfrakturen auch als pathologische Frakturen bei benignen (z. B. juve-
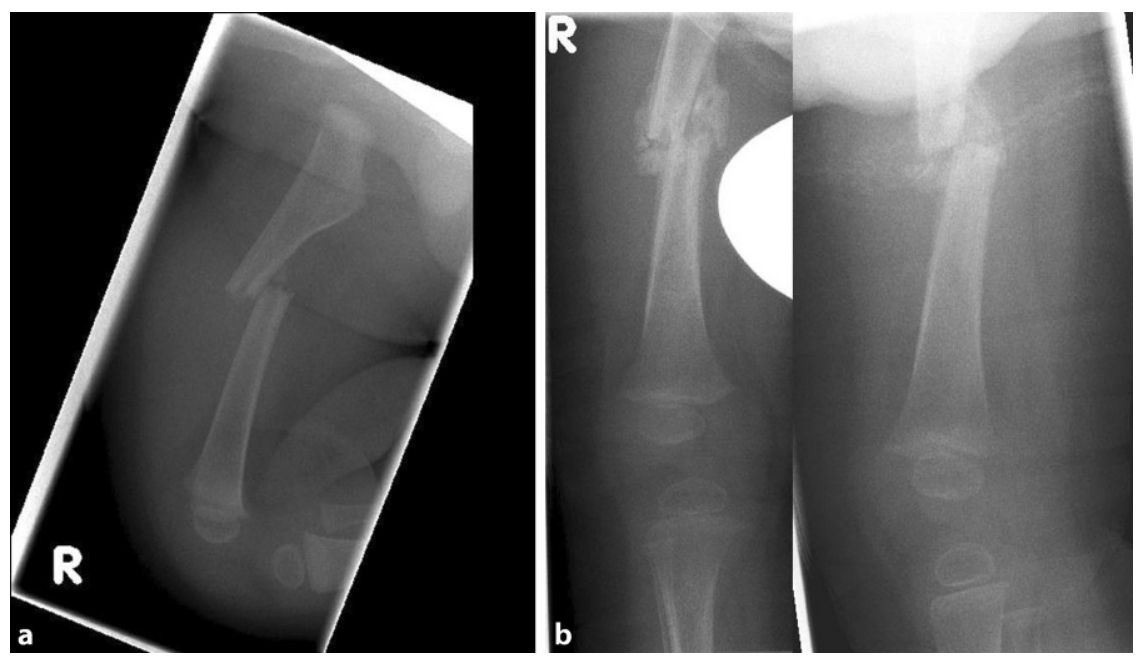

Abb. 1 A Konservative Behandlung einer misshandlungsbedingten Femurschaftfraktur mit Overheadextension bei einem 7 Monate alten Kind. Nach $21 / 2$ Wochen zeigt sich bereits eine ausreichende Konsolidierung. a Röntgen am Aufnahmetag, b Konsolidation nach 2 1/2 Wochen in der Overhead-Extension

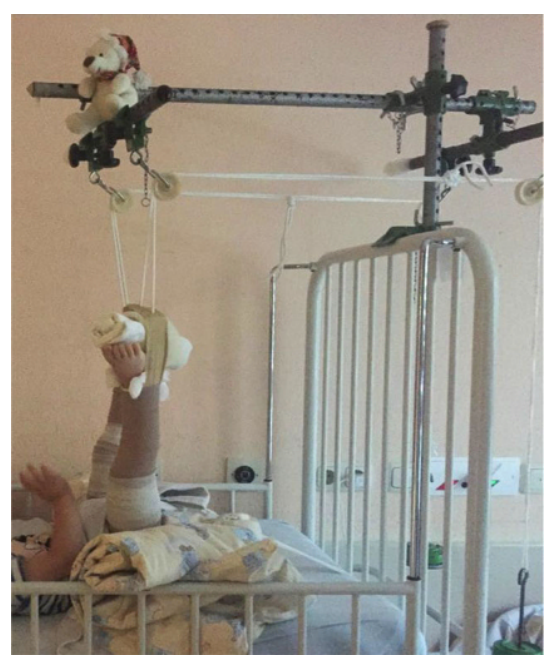

Abb. 2 \& Overheadextension bei einem 2-jährigen Kind 


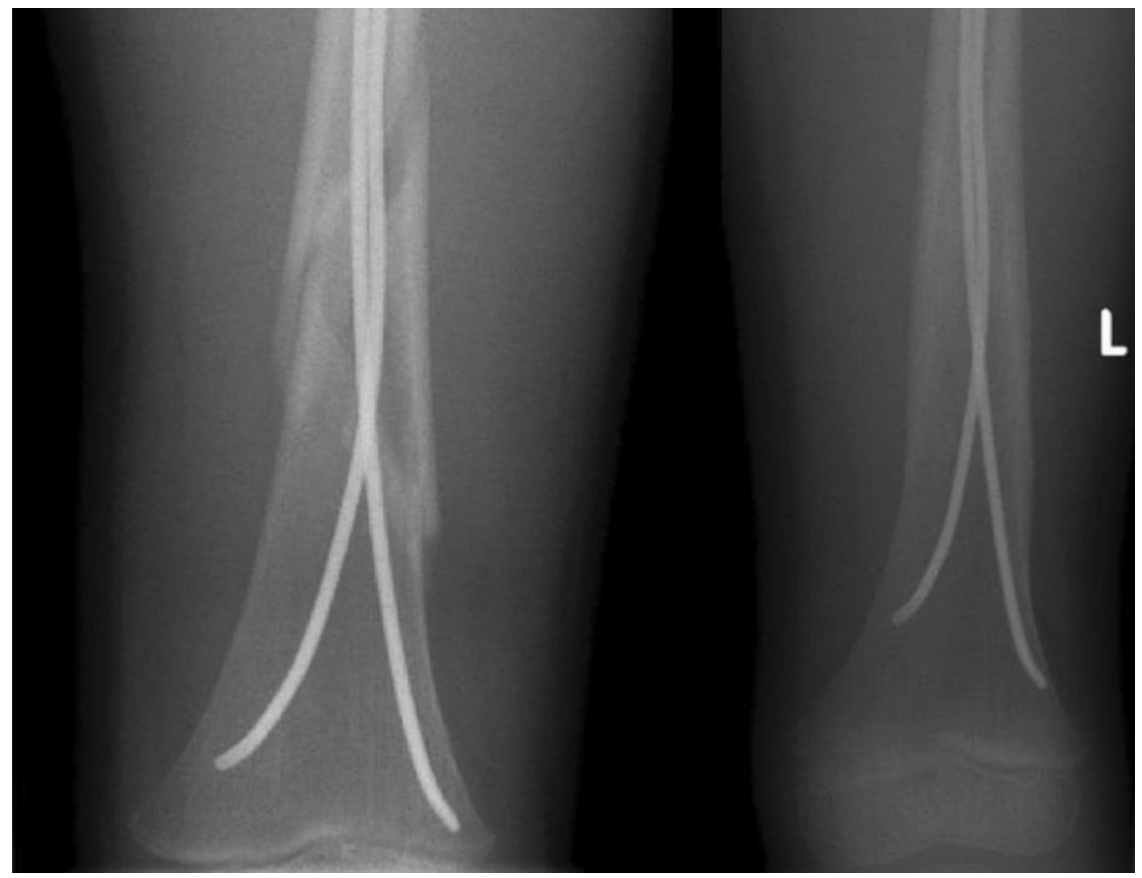

Abb. $3 \Delta$ AntegradeESIN (elastisch stabile intramedulläre Nagelung)-Osteosynthese bei einem 5-jährigen Jungen mit Femurspiralfraktur im distalen Drittel nile Knochenzyste) und malignen Knochenerkrankungen vor.

\section{Wachstumsprognose}

Wie überall im kindlichen Skelett führt der Knochen im Rahmen der Frakturheilung unterschiedliche Umbauvorgänge (Remodeling) durch.

Hierbei werden Seit-zu-Seit-Verschiebungen einwandfrei korrigiert, und auch Varusfehlstellungen erfahren insbesondere bei sehr jungen Kindern und entsprechend viel verbleibendem Wachstum eine gewisse Korrektur. Daneben werden Valgus- und Rekurvationsfehlstellungen nur unzureichend korrigiert [7, S. 298-314].

Die Frage nach der Korrektur verbliebener Rotationsfehlstellungen ist ein häufig diskutiertes Thema und kann nach der aktuellen Studienlage nicht abschließend beantwortet werden. Da es bis etwa zum 10. Lebensjahr auch physiologischerseits zu einer Detorsion des Schenkelhalses während des Längenwachstums kommt - die Schenkelhalsantetorsion beträgt bei Geburt $40-50^{\circ}$ und reduziert sich auf $10-15^{\circ}-$, scheint dies auch eine Korrektur einer

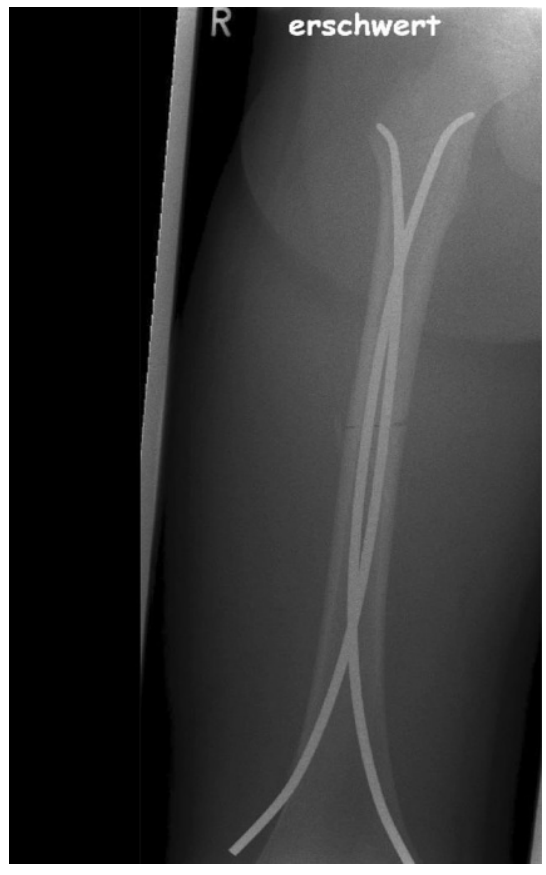

Abb. 4 ム Korrekte Platzierung der Nägel bei der retrograden Technik. Beide Nägel füllen gemeinsam zwei Drittel des Markraumes aus und spannen sich auf Frakturhöhe auf

\section{Diagnostik}

fehlstellung zu ermöglichen. Allerdings ist diese Form des Remodelings nicht ausreichend zuverlässig, als dass es in unser Therapiekonzept integriert werden könnte. Andererseits kommen asymmetrische Rotationsverhältnisse des Femurs auch physiologisch vor und sind bis zu einem Ausmaß von $25^{\circ}$ [3] klinisch irrelevant.

Wachstumsstörungen hemmender Art, also vorzeitige Verschlüsse der Femurfrakturen keine Rolle, während es obligat zu einer Stimulation des Wachstums durch die Fraktur kommt.

Wie auch an anderen Lokalisationen im wachsenden Skelett ist das Ausmaß der Wachstumsstimulation umso größer, je mehr Remodeling stattfinden muss [7, S. 52-54]. Das heißt, je mehr Achsabweichung im Rahmen der primären Frakturbehandlung verblieben ist und je länger die Heilung des Knochens dauert, desto ausgeprägter ist die zu erwartende Verlängerung der betroffenen Extremität.

Eine gründliche Anamnese des Unfallmechanismus spielt insbesondere bei Kindern in den ersten 3 Lebensjahren eine wesentliche Rolle, da sich hier bereits erste Verdachtsmomente einer Kindesmisshandlung ergeben können. Daneben stellen die Inspektion der betroffenen Extremität und die Untersuchung der peripheren Durchblutung, Motorik und Sensibilität die einzigen klinischen Untersuchungen dar.

Das konventionelle Röntgenbild in 2 Ebenen ist die Standardbildgebung bei Femurschaftfrakturen im Kindesalter [8]. Hiervon kann abgewichen werden, wenn sich bereits in der ersten angefertigten Ebene die Indikation zur operativen Therapie ergibt.

Eine ausgedehntere Diagnostik ist bei Verdacht auf Kindesmisshandlung oder bei pathologischen Frakturen nach den entsprechenden Leitlinien indiziert.

\section{Therapie} Wachstumsfuge, spielen bei diaphysären

Im Wesentlichen wird die Entscheidung zwischen konservativer und operativer Therapie nicht aufgrund des Frakturtyps 
oder des Dislokationsausmaßes, sondern aufgrund des Alters des Patienten getroffen [9].

\section{Kinder bis zum 3. Lebensjahr}

Nach der aktuellen Leitlinie zur Behandlung kindlicher Femurschaftfrakturen [8] und den gängigen kindertraumatologischen Lehrbüchern werden Oberschenkelfrakturen bei Kindern jünger als 3 Jahre prinzipiell konservativ behandelt.

Grundlagen dieser Therapieentscheidung sind das hohe Remodelingpotenzial und die schnelle Konsolidierung (- Abb. 1) in dieser jungen Altersklasse, wenn auch eine potenzielle stimulative Wachstumsstörung durch die verbliebenen Achsabweichungen in Kauf genommen wird.

Prinzipiell stehen für die konservative Therapie 2 Methoden zur Verfügung:

- die Overheadextension und

- der Becken-Bein-Gips.

Die Wahl der Methode obliegt dem behandelnden Arzt und basiert meist auf der vorhandenen Kliniklogistik und persönlichen Erfahrung. Vergleichende Studien der beiden Methoden liegen nicht vor.

Die Overheadextension wird in der Regel in Analgosedierung oder kurzer Narkose als Pflasterextension angelegt. Im Anschluss wird das betroffene Bein mit einem Fünftel bis einem Sechstel des Körpergewichtes extendiert (• Abb. 2).

Die Dauer dieser Behandlung beträgt je nach Alter des Kindes 2 bis 3 Wochen und wird stationär durchgeführt.

Der Becken-Bein-Gips wird ebenfalls in Sedierung oder Narkose angelegt. In der Regel erfolgt auf der frakturierten Seite ein Fußeinschluss, um zumindest partiell eine Kontrolle der Rotation zu erreichen, während auf der gesunden Seite ein Gips bis proximal des Kniegelenkes ausreichend ist. Der Becken-BeinGips kann aus Kunststoffcast angefertigt werden. Dies reduziert das Gewicht und macht die Versorgung im Vergleich zum klassischen Weißgips weniger anfällig für Verschmutzungen und Nässe.

Bei beiden Verfahren sind radiologische Kontrollen während der Therapie nicht zwingend erforderlich, eine Kon-

Trauma Berufskrankh 2018 20 (Suppl 1):S1-S5 DOI 10.1007/s10039-017-0269-3

(c) Springer Medizin Verlag GmbH 2017

M. Kertai

\section{Behandlung der Femurschaftfraktur im Wachstumsalter. Vom Beckenbeingips bis zur elastisch stabilen intramedullären Nagelung}

\section{Zusammenfassung}

Femurschaftfrakturen im Kindesalter zeigen im Wesentlichen 2 Altersgipfel. Der eine betrifft Kinder im Alter zwischen 2 und 4 Jahren, der zweite findet sich um die Pubertät. Während in der Gruppe der Kleinkinder Femurschaftfrakturen bereits bei relativ geringer Kraftweinwirkung vorkommen, handelt es sich beim Unfallmechanismus bei den älteren Kindern meist um Hochrasanztraumen. Bei noch nicht gehfähigen Kindern muss stets eine Kindesmisshandlung als Ursache ausgeschlossen werden. Die Behandlung der Femurschaftfraktur im Alter bis 3 Jahre ist in der Regel konservativ mittels Becken-BeinGips oder Overheadextension, bei älteren Kindern ist dies die Domäne der elastisch stabilen intramedullären Nagelung (ESIN).

Schlüsselwörter

Kindesmisshandlung · Kind · Unfall · Hochrasanztrauma. Gips

\section{Treatment of femoral shaft fractures in the growing phase. From hip spica casts to elastic stable intramedullary nailing}

\begin{abstract}
There are two essential age peaks in pediatric femoral shaft fractures. The first peak concerns children between the ages of 2-4 years and the second peak is around puberty. In young children femoral shaft fractures may occur due to a relatively minor accident but the accident mechanism in older children usually involves high-velocity trauma. In children that are not yet at the walking stage, child abuse must always be excluded as the cause of the
\end{abstract}

fracture. Treatment of femoral shaft fractures up to the age of 3 years is usually conservative by means of a cast or extension therapy, whereas in older children this is the domain of elastic stable intramedullary nailing (ESIN).

\section{Keywords}

Child abuse $\cdot$ Child $\cdot$ Accident $\cdot$ High velocity trauma · Plaster casts solidationskontrolle nach 14 bzw. 21 Tagen wird jedoch empfohlen. Ebenso sollte eine Kontrolle in der ersten Woche nach Therapiebeginn erfolgen, wenn ein Therapiewechsel daraus resultieren könnte.

Bereits in der Leitlinie [8] wird darauf hingewiesen, dass im „Einzelfall [die operative Therapie] auch bei Kindern unterhalb des 3. Lebensjahres angewendet werden“ kann. In einer Umfrage der Deutschen Gesellschaft für Unfallchirurgie zeigte sich, dass die Hälfte aller teilnehmenden Behandler in Deutschland auch bei Kindern vor dem 3. Lebensjahr eine operative Therapie durchführt [10]. Da hierzu jedoch keine Evidenz vorliegt, ist eine entsprechende prospektive Studie durch die Sektion Kindertraumatologie der Deutschen Gesellschaft für Unfallchirurgie in Arbeit.
Nichtsdestotrotz steht nach wie vor die konservative Therapie bei den Kleinkindern aufgrund der schnellen Konsolidation und des Remodelings klar im Vordergrund.

Aus Sicht des Autors kann die operative Therapieoption bei bereits gehfähigen Patienten mit dessen Eltern im Sinne der umfassenden Aufklärung diskutiert werden. Mögliche Gründe für die operative Therapiealternative stellen insbesondere soziale Faktoren sowie potenziell klinisch relevante stimulative Wachstumsstörungen und eine Kontrolle über die Rotationsverhältnisse dar.

\section{Kinder älter als 3 Jahre}

Ab dem 3. Lebensjahr stellt die elastisch stabile intramedulläre Nagelung (ESIN) die Therapie der Wahl dar. Diese wird 

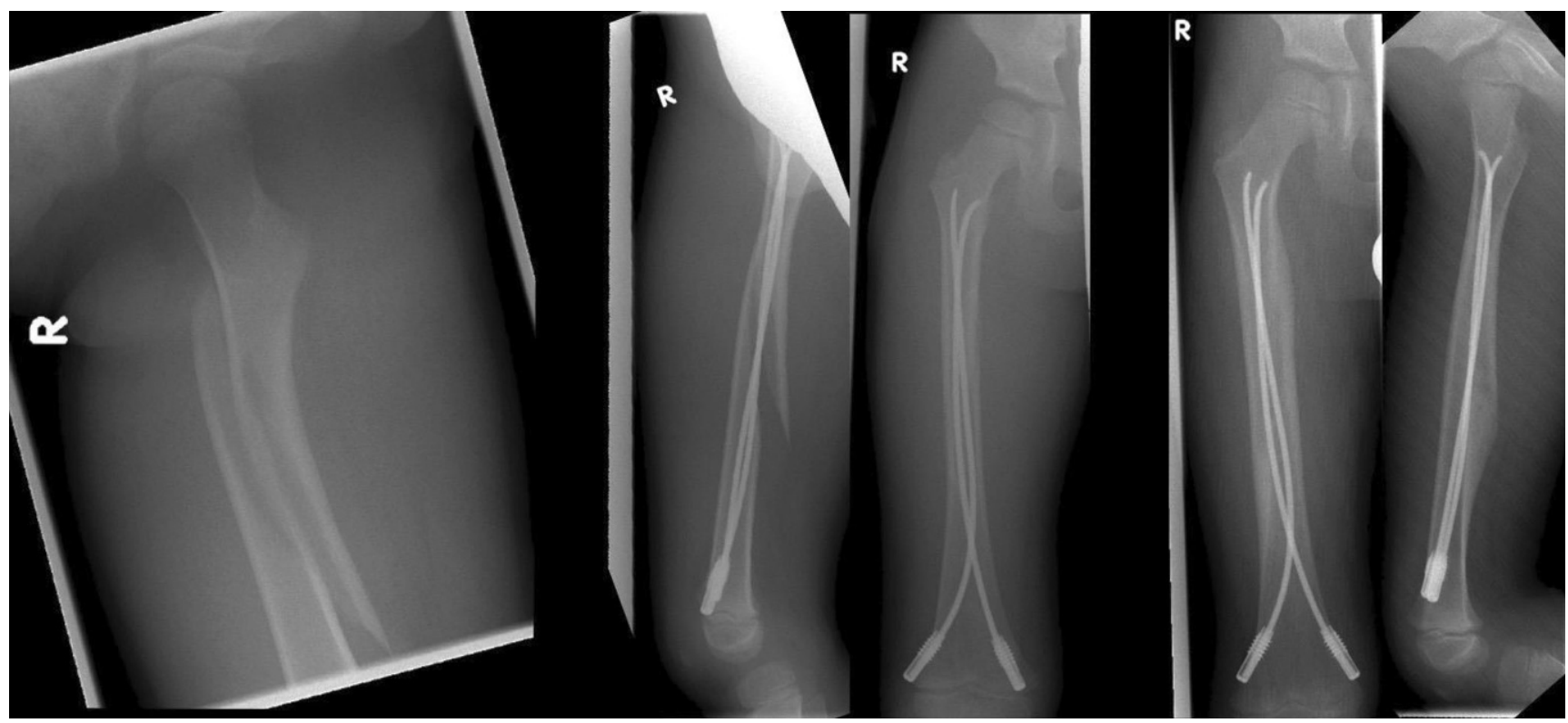

Abb. 5 A Versorgung einer längsinstabilen Femurfraktur bei einem 6-jährigen Mädchen unter Verwendung von Verriegelungsschraubkappen

bei Frakturen im proximalen und mittleren Drittel antegrad, im distalen Drittel retrograd (๑ Abb. 3) durchgeführt [11].

Bei der Durchführung der ESIN-Osteosynthese ist auf die Wahl des korrekten Nagedurchmessers (ein Drittel des Markraumdurchmessers je Nagel) und einer guten Aufspannung in Frakturhöhe zu achten ([11, 12]; - Abb.4). Ideal eignet sich die ESIN-Osteosynthese bei Quer- oder kurzen Schrägfrakturen.

Kann bei längeren Querfrakturen oder Mehrfragmentfrakturen durch die ESIN-Osteosynthese keine ausreichende Längsstabilität erreicht werden, so kann dies durch die Verwendung von Verriegelungsschraubkappen erreicht werden. Die Verwendung einer distalen Verriegelung, z.B. End-Cap (• Abb. 5), ermöglicht jedoch auch bei längsinstabilen Frakturen in vielen Fällen eine sichere Osteosynthese. Diese Modifikation wurde sowohl biomechanisch als auch klinisch evaluiert [13, 14].

Radiologische Kontrollen werden an den ersten postoperativen Tagen, sofern keine adäquaten Durchleuchtungsbilder vorhanden sind, nach 4 Wochen und nach 3 bis 6 Monaten vor der Metallentfernung empfohlen [8]. Zur Reduktion des Refrakturrisikos wird empfohlen, die Metallentfernung erst 6 Monate nach der Frakturversorgung durchzuführen.

\section{Nachbehandlung}

Völlig unabhängig von der primären Therapie muss bei allen Kindern mit stattgehabter Femurschaftfraktur eine adäquate klinische Nachkontrolle erfolgen. Diese wird erstmalig zum Zeitpunkt der Konsolidationskontrolle durchgeführt und wird anschließend halbjährlich, mindestens jedoch jährlich für weitere 2 Jahre fortgesetzt. Die Nachkontrollen beinhalten die klinische Kontrolle der Beinachse, der Rotationsverhältnisse im Seitenvergleich sowie der Beinlänge bzw. des Beckenstandes im Stehen. Ergeben sich hier Auffälligkeiten, so müssen die Nachkontrollen jährlich bis zum Wachstumsabschluss erfolgen. Radiologische Kontrollen sind nur bei Auffälligkeiten und bei Planung einer Therapie notwendig.

\section{Fazit für die Praxis}

- Bei Femurschaftfrakturen noch nicht frei gehfähiger Kinder muss stets an eine Kindesmisshandlung als Ursache gedacht und diese ausgeschlossen werden.

- Femurschaftfrakturen kommen bei Vorschulkindern meist als Monotrauma, im höheren Alter häufig im
Rahmen von Hochrasanztraumen mit Begleitverletzungen vor.

- Die Therapie von Femurschaftfrakturen bis zum Alter von 3 Jahren kann konservativ erfolgen.

- Ab dem Alter von 3 Jahren ist die Behandlung kindlicher Femurschaftfrakturen die Domäne der elastisch stabilen intramedullären Nagelung.

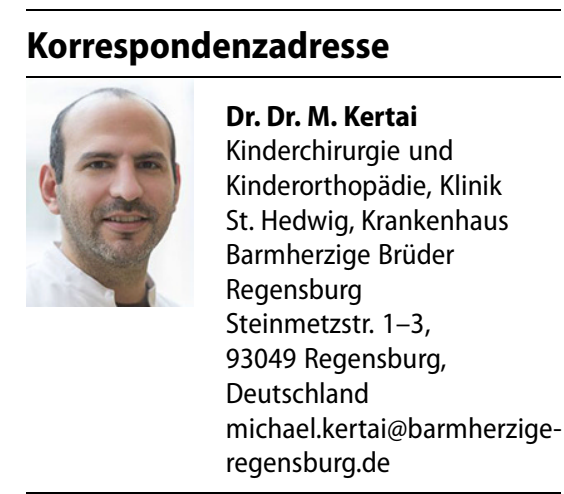

\section{Einhaltung ethischer Richtlinien}

Interessenkonflikt. M. Kertai gibt an, dass kein Interessenkonflikt besteht.

Dieser Beitrag beinhaltet keine vom Autor durchgeführten Studien an Menschen oder Tieren.

The supplement containing this article is not sponsored by industry. 


\section{Literatur}

1. Kraus R,SchneidmüllerD, RöderC (2005)Häufigkeit von Frakturen der langen Röhrenknochen im Wachstumsalter.Dtsch Ärztebl 12:838-842

2. Landin LA (1983) Fracture patterns in children. Analysis of 8682 fractures with special reference to incidence, etiology and secular changes in a Swedish urban population1950-1979. Acta Orthop Scand Suppl 202:1-109

3. Schlickewei W, Seifel Nasr M, Weinberg A-M (2006) Diaphysärer Oberschenkel. In: Weinberg A-M, Tscherne $\mathrm{H}$ (Hrsg) Unfallchirurgie im Kindesalter. Springer, Berlin, S636-671

4. Jawadi AH, Letts M (2003) Injuries associated with fractures of the femur secondary to motor vehicle accidents in children. Am J Orthop 32:459-462

5. Maguire S, Cowley L, Mann M, Kemp A (2013) What does the recent literature add to the identification and investigation of fractures in child abuse: an overview of reviewupdates 2005-2013. Evid Based Child Health 8:2044-2057

6. Flynn JM, Skaggs DL (2014) Femoral Shaft Fractures. In: Flynn JM, Skaggs DL, Waters PM (Hrsg) Rockwood and Wilkins' Fractures in Children. Wolters Kluwer, Philadelphia, S987-1026

7. von Laer L, Kraus R, Linhart WE (2012) Frakturen und Luxationen im Wachstumsalter. Thieme, Stuttgart

8. S1-Leitlinie 006/016: Femurschaftfraktur im Kindesalter. Stand 09/2014

9. Wright JG, Wang EE, Owen JL, Stephens D, Graham HK, Hanlon M, Nattrass GR, Reynolds RA, Coyte P (2005) Treatment of paediatric femoral fractures: a randomised trial. Lancet 365:1153-1158

10. Strohm PC, Schmittenbecher PP (2015) Femoral shaft fractures in children under 3 years old.Current treatment standard. Unfallchirurg 118(1):48-52

11. Metaizeau JP (2004) Stable elastic intramedullary nailing for fractures of the femur in children. J Bone Joint Surg Br 86:954-957

12. Lascombes P, Huber H, Fay R, Popkov D, Haumont T, Journeau P (2013) Flexible intramedullary nailing in children: nail to medullary canal diameters optimal ratio. J Pediatr Orthop 33:403-408

13. Slongo T, Audigé L, Hunter JB, Berger SM (2011) Clinical evaluation of end caps in elastic stable intramedullary nailing of femoral and tibial shaft fractures in children. Eur J Trauma Emerg Surg 37:305-312

14. KaiserMM,ZachertG, WendlandtR, RappM, Eggert C, Stratmann C, Wessel LM, Schulz AP, Kienast B (2011) Biomechanical analysis of a synthetic femoral spiral fracture model: Do end caps improve retrograde flexible intramedullary nail fixation? J Orthop Surg Res 6:46 